\title{
Expanded study on the risk of lymphovascular space invasion and lymph node metastasis of endocervical adenocarcinoma using Pattern Classification: a single- centre analysis of 213 cases
}

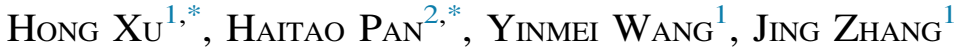 \\ ${ }^{1}$ State Key Laboratory of Cancer Biology, Department of Pathology, Xijing Hospital and \\ School of Basic Medicine, Fourth Military Medical University, Xi'an, Shaanxi, China; \\ ${ }^{2}$ Department of Biostatistics, St Jude Children's Research Hospital, Memphis, TN, USA; \\ * these authors contributed equally to this work
}

\section{Summary}

A new three-tiered Pattern Classification system for usualtype endocervical adenocarcinomas (U-EACs) recommends using tumour invasive patterns rather than depth of invasion (DOI) and horizontal spread to categorise tumours. Tumours categorised by Pattern Classification are associated with lymph node (LN) metastasis and adverse outcomes. The aim of this study is to further explore the potential of Pattern Classification in surgical pathology practice. A total of 213 consecutive cases [201 U-EACs and 12 gastric-type adenocarcinomas (GACs)] diagnosed between 2006 and 2017 was retrospectively analysed. Clinicopathological data included age at diagnosis, DOI measurement, the status of lymphovascular space invasion (LVSI) and LN metastasis, and the number of LVSI foci, dissected and metastatic LNs. Immunostaining for CD34 and D2-40 was performed to identify LVSI in 14 challenging cases. Overall, mean age at diagnosis was 51 years (range 23-75). LVSI and LN metastasis occurred in $128(60.1 \%$, $128 / 213)$ and $42(20.5 \%, 42 / 205)$ cases, respectively. Also, $28(13.1 \%), 21(9.9 \%)$, and $164(77.0 \%)$ patients had pattern $\mathrm{A}, \mathrm{B}$, and $\mathrm{C}$ tumours, respectively. Patients with pattern $\mathrm{C}$ tumours had the oldest age at diagnosis $(p=0.007)$, the highest incidence of LVSI and LN metastasis, and the highest DOI $(p<0.001)$. Due to a highly heterogeneous growth pattern, pattern $\mathrm{C} U$-EACs were stratified into four subgroups: C1, C2 and C3 corresponded to solid, extensive linear destructive, and band-like lymphocytic infiltrate growth patterns, respectively, and C4 included diffuse destructive, confluent, micropapillary and mixed growth pattern. C2 and C3 subgroup tumours had lower incidence of LVSI (20\% and $40 \%$, respectively) than the other two subgroups ( $p<0.001)$. None of the patients with $\mathrm{C} 2$ and $\mathrm{C} 3$ subgroup had LN metastasis and $\geq 3 \mathrm{LVSI}$ foci. All GACs belonged to pattern $\mathrm{C}$ and had deeper stromal invasion $(p=0.008)$, higher incidence of LN metastasis ( $p=0.001)$, and larger quantity of LVSI foci $(p=0.008)$ and metastatic LNs $(p=0.004)$ than those of pattern C U-ECAs. Number of LVSI foci were moderately positively correlated with LN metastasis status $(p<0.001$, $\gamma=0.489)$ or number of metastatic LNs $(p<0.001, \gamma=0.409)$. Our study further supports that Pattern Classification is a system easy to follow, which has a strong correlation to LVSI and an effective predictability for LN metastasis. Extensive
\end{abstract}

linear destructive and band-like lymphocytic infiltrate growth patterns in pattern $\mathrm{CU}$-EACs need to be recognised, as they behave less aggressively than that for the other growth pattern subgroups. Our study supports that Pattern Classification can be routinely applied to guide therapies for patients with U-EACs.

Key words: Endocervical adenocarcinoma; invasive pattern; growth pattern; lymph node metastasis; lymphovascular space invasion.

Received 19 February, revised 21 April, accepted 28 April 2019 Available online 21 August 2019

\section{INTRODUCTION}

Adenocarcinoma accounts for $20-25 \%$ of all cervical carcinomas, with recent incidence data indicating an upward trend. ${ }^{1}$ Although nearly 10 histological types are listed in the 2014 World Health Organization (WHO) classification, ${ }^{2}$ almost all adenocarcinomas of the cervix (ADCs) can be grouped into two categories on the basis of high-risk human papillomavirus (HPV) infection, which can be recognised by the presence or absence of luminal mitoses and apoptosis seen at scanning magnification. ${ }^{3}$ HPV-related adenocarcinomas constitute more than $80 \%$ of ADCs, with the vast majority being usualtype endocervical adenocarcinomas (U-EACs). ${ }^{4}$ Non-HPVrelated adenocarcinomas primarily consist of mucinous adenocarcinomas, gastric type (so-called gastric-type adenocarcinomas, GACs). The exact incidence of GACs is unclear because of their previous misclassification. ${ }^{5}$ Stolnicu et al. reported that GACs are the second most common subtype of adenocarcinomas in the cervix and comprise $10 \%$ of all adenocarcinomas. ${ }^{3}$ It is reported that GACs have a more aggressive clinical course and are associated with a remarkably decreased 5-year disease-specific survival and higher risk for disease recurrence than for U-EACs. ${ }^{6,7}$

Numerous clinicopathological studies have shown that in routine practice, evaluation of invasive ADCs poses diagnostic challenges such as correctly diagnosing invasive adenocarcinoma and precisely measuring the depth of invasion (DOI). ${ }^{4}$ The former involves the identification of welldifferentiated and/or superficially invasive U-EACs from adenocarcinoma in situ (AIS) and GACs (especially well- 
differentiated variant minimal deviation adenocarcinoma) from benign mucinous endocervical glandular proliferations. ${ }^{4}$ The latter includes the identification of a specific, highly reproducible reference point to measure DOI in an early carcinoma. Given that an accurate DOI measurement is essential for the International Federation of Gynecology and Obstetrics (FIGO) staging system, ${ }^{8}$ attention needs to be focused on solving these challenges.

A recent series of studies has documented a three-tiered pattern-based system (Pattern Classification; also called Silva Classification) to classify U-EACs. ${ }^{9-11}$ This novel classification system recommends the use of tumour invasive patterns rather than traditional measurement of DOI and horizontal spread to categorise tumours. According to this classification, U-EACs are divided into patterns A, B, and C. Notably, there is a close correlation among different patterns and tumour biological behaviours. Pattern Classification can yield a more accurate predicted value of lymph node (LN) metastasis and tumour recurrence than FIGO staging does. $^{9-11}$ Accordingly, different treatment strategies have been suggested on the basis of this classification. ${ }^{11}$

To further explore the potential of Pattern Classification in clinical application, we retrospectively analysed 201 cases of U-EAC and further categorised pattern $\mathrm{C}$ tumours into four growth pattern subgroups as described previously. ${ }^{12}$ In addition, we extended our study to include 12 cases of the uncommon GAC. We investigated the correlation among the three invasive patterns and clinicopathological variables, and determined the clinical significance in four growth pattern subgroups of U-EAC with pattern $\mathrm{C}$. We also compared the difference between U-EAC with pattern $\mathrm{C}$ and GAC, and highlighted the relationship between lymphovascular space invasion (LVSI) and LN metastasis.

\section{MATERIALS AND METHODS}

\section{Patients}

In this retrospective study, records of all histologically diagnosed patients with invasive ADC who underwent surgery between December 2006 and March 2017 were retrieved from files in the Department of Pathology at Xijing Hospital. The 224 patients who did not receive neoadjuvant chemotherapy and/or radiotherapy underwent complete tumour resection by the cone/loop electrosurgical excision procedure (2 cases), or hysterectomy with pelvic LN dissection (216 cases) or without pelvic LN dissection (6 cases) Entire tumour tissues were submitted for microscopic examination to evaluate their patterns. The margins were confirmed to be negative in all cone/loop electrosurgical excision procedure specimens. All slides with haematoxylin and eosin (H\&E) and immunohistochemical (IHC) staining were reviewed simultaneously by two of the authors ( $\mathrm{HX}$ and $\mathrm{JZ}$ ) at the time of retrospective analysis. Diagnostic criteria for ADC histological types were established as per the 2014 WHO classification ${ }^{2}$ and the International Endocervical Adenocarcinoma Criteria and Classification (IECC) system. ${ }^{3,13}$ For this study, 201 cases of U-EAC and 12 cases of GAC were included; the remaining 11 cases of other histological types of ADCs (clear cell carcinoma, 7 cases; mesonephric carcinoma, 1 case; serous carcinoma, 1 case; endometrioid carcinoma, 2 cases) were excluded. This study was approved by the Institutional Ethics Board at Xijing Hospital. Informed consents were signed by all participants.

\section{Pattern classification}

The 201 cases of U-EAC and 12 cases of GAC were classified by Pattern Classification. ${ }^{9-11}$ Pattern A tumours had no destructive stromal invasion and LVSI and were characterised by well-defined glands with rounded contours, sometimes having a relatively well-preserved lobular architecture (Fig. 1). Pattern B tumours had focal destructive stromal invasion arising from pattern A-like glands, which exhibited individual or small clusters of tumour cells or fragments of glands within a reactive (desmoplastic, oedematous, or inflammatory) stroma (Fig. 2). Pattern C tumours typically showed diffusely destructive stromal invasion, which manifested as diffusely infiltrative glands, solid or poorly differentiated component within an extensively diffused desmoplastic stroma, or confluent growth filling a $4 \times$ field $(5 \mathrm{~mm})$.

Recently, six architectural growth patterns - diffuse destructive, confluent, extensive linear destructive, band-like lymphocytic infiltrate, solid, and micropapillary - were summarised by Alvarado-Cabrero et al. ${ }^{12}$ On the basis of that study and results from our current study, we further stratified pattern C tumours into four subgroups: (1) $\mathrm{C} 1$, more than $50 \%$ of the tumour component displayed a solid growth pattern, defined by a poorly differentiated component with sheet, cord, or nest architecture and lacking prominent glandular differentiation (Fig. 3A,B); (2) C2, tumours showed an extensive linear destructive growth pattern, which was composed of diffuse laminar malignant cells $>5 \mathrm{~mm}$ in size, and invasion only at the base of the tumour and parallel to the surface (Fig. 3C); (3) C3, tumours with band-like lymphocytic infiltrate growth pattern exhibited a prominent lymphoid infiltration in the neoplastic glandular elements (Fig. 3D); and (4) C4, diffuse destructive, micropapillary, confluent, and mixed growth pattern (the minimum percentage of the second growth pattern was set at $10 \%$ ). Tumours presented as extensively disordered angulate and open glands (diffuse destructive growth pattern, Fig. 3E); micropapillary growth pattern (Fig. 3F); densely packed glands with cribriform or papillae filling a $4 \times$ or $5 \mathrm{~mm}$ field (confluent growth pattern, Fig. 3G,H), or mixed at least two different growth patterns.

\section{Data collection}

The following parameters were collected and recorded: age at diagnosis; DOI measurement; the status (presence or absence) of LVSI and LN metastasis; and the number of LVSI foci, dissected and metastatic LNs. DOI was measured from gland crypts of the adjacent non-invasive component to the deepest point of invasion. The status of LVSI and LNs were assessed independently, without knowledge of the latter when the former was assessed. LVSI was defined as a single or small cluster of malignant cells in spaces with covering endothelium. Some tumour cells had inverted polarity, dense eosinophilic cytoplasm, or coexisted with pink proteinaceous material in the

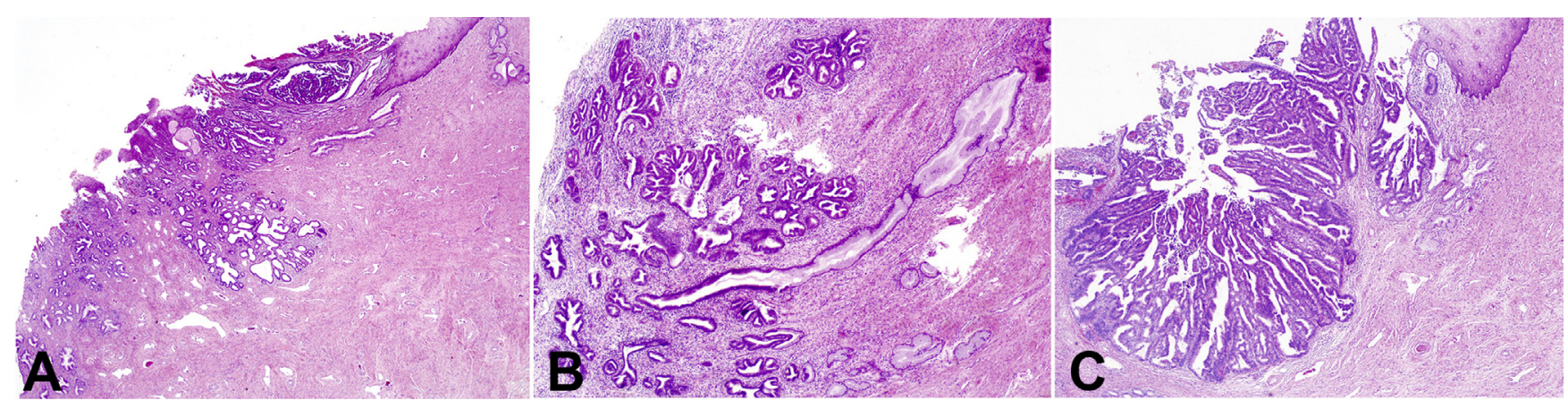

Fig. 1 Pattern A tumours. This tumour is present as papillary intraglandular and demonstrates a pushing pattern of invasion without stromal invasion (A). Tumours have a preserved lobular architecture $(\mathrm{B}, \mathrm{C})$. 


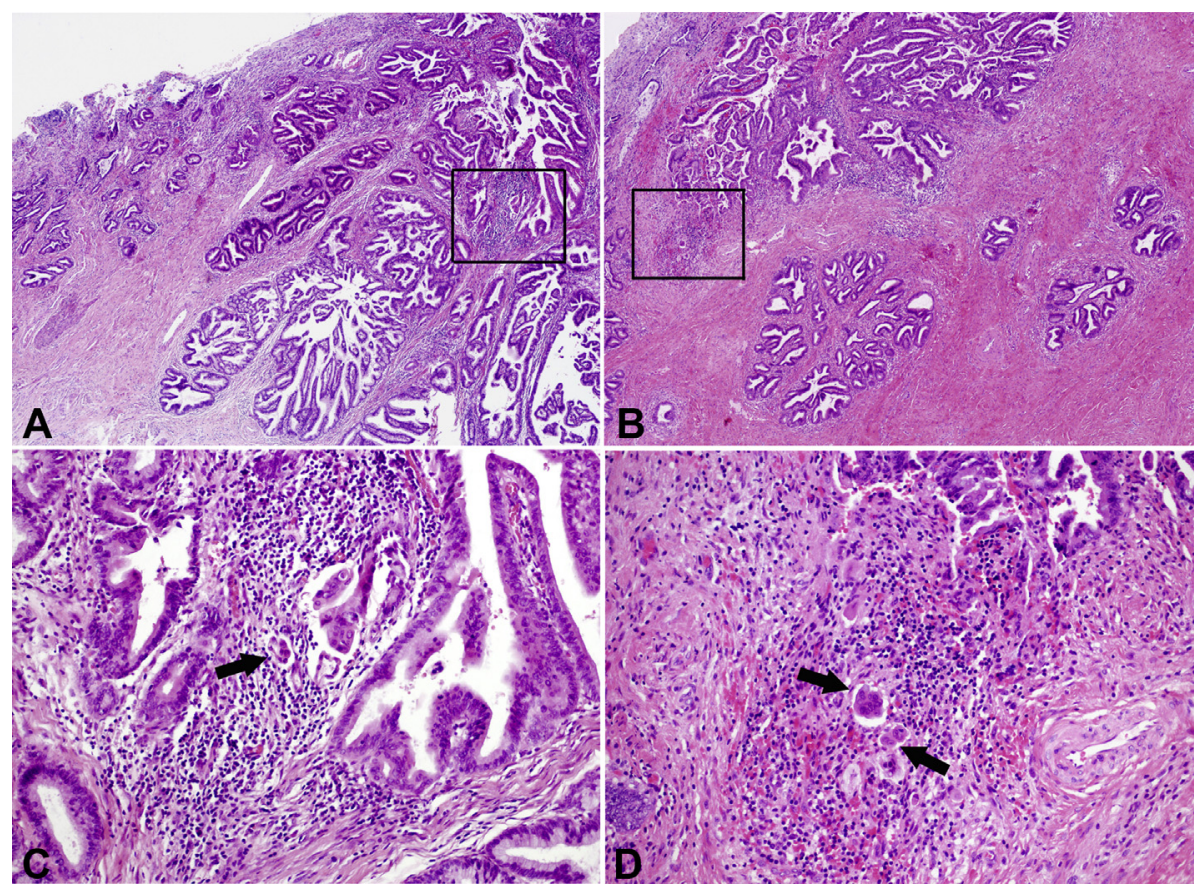

Fig. 2 Pattern B tumours. These tumours show early destructive stromal invasion arising from pattern A. At low magnification, small foci of invasion are too subtle to be easily detected (A,B). However, focal or multifocal cell clusters with inflammatory reaction can be seen at high magnification (C,D). These invasive tumour cells often have eosinophilic cytoplasm (black arrows).

lumen. In 14 cases, IHC analysis was performed for CD34 and D2-40 (Dako, Denmark) to ascertain true LVSI. Positive staining for CD34 only was interpreted as blood vessels. Positive expression of both D2-40 and CD34 was interpreted as lymphatic vessels.

\section{Statistical analyses}

All analyses were performed using SPSS statistical software package (version 16.0; IBM, USA). Analysis of variance (ANOVA), Student's $t$ tests, or chisquared test were used to compare categorical variables. Correlation coefficients were analysed by Pearson correlation. A $p$ value less than 0.05 was considered statistically significant. All statistical tests were two sided.

\section{RESULTS}

A total of 213 patients [mean age at diagnosis 51 years (23-75 years)] with invasive adenocarcinoma (201 U-EACs and 12 GACs) were analysed by Pattern Classification. Overall, LVSI and LN metastasis occurred in $128(60.1 \%$, $128 / 213)$ and $42(20.5 \%, 42 / 205)$ patients, respectively. There were $28(13.1 \%)$ patients with pattern A tumours, 21 (9.9\%) with pattern B tumours, and 164 (77.0\%) with pattern C tumours. Notably, all GACs were pattern C tumours. Clinical and pathological parameters are shown by pattern type in Table 1 .

Mean age at diagnosis for patients with pattern A tumours was 43 years (range 26-66 years). Twenty-four patients received hysterectomy with pelvic LN dissection, two underwent hysterectomy without pelvic LN dissection, and two were treated by the cone/loop electrosurgical excision procedure. The majority of tumours $(71.4 \%)$ had $<3 \mathrm{~mm}$ DOI. No patient had the presence of LVSI and LN metastasis. Mean age at diagnosis for patients with pattern B tumours was 48 years (range 34-67 years). The patients underwent hysterectomy with pelvic LN dissection (18 cases) or without pelvic LN dissection (3 cases). All 18 patients were free of LN metastasis. Five patients had LVSI. Mean age at diagnosis for patients with pattern $\mathrm{C}$ tumours was 49 years (range
23-75 years). The patients were treated by hysterectomy with pelvic LN dissection (163 cases) or without pelvic LN dissection (1 case). Incidences of LVSI and LN metastasis were $75.0 \%$ (123/164) and $26.1 \%$ (42/163), respectively. More than $60 \%$ of patients had $\geq 3$ LVSI foci $(61.5 \%, 80 /$ $164)$ and $>10 \mathrm{~mm}$ DOI $(64.6 \%, 106 / 164)$. Among the three invasive patterns, patients with pattern $\mathrm{C}$ tumours had the oldest age at diagnosis $(p=0.007)$ and the highest incidence of LVSI $(p<0.001)$, LN metastasis $(p<0.001)$, and $>10 \mathrm{~mm}$ DOI $(p<0.001)$. Patients with pattern A tumours had the youngest age at diagnosis, and none of them had LVSI, LN metastasis, and $>10 \mathrm{~mm}$ DOI.

To better understand the different architectural growth patterns in U-EACs with pattern C, 152 patients were further stratified into four growth pattern subgroups. There were 13 (8.5\%) patients with $\mathrm{C} 1$ subgroup tumours, 15 (9.9\%) patients with $\mathrm{C} 2$ subgroup tumours, five $(3.2 \%)$ patients with C3 subgroup tumours, and $119(78.3 \%)$ patients with C4 subgroup tumours. The four subgroups are compared in Table 2. Briefly, patients with C2 subgroup tumours, which exhibited extensive linear destructive growth pattern, had the lowest incidence of LVSI $(20.0 \%, p<0.001)$ among the four growth pattern subgroups. All patients had $<3$ LVSI foci $(p=0.033)$ and $\leq 10 \mathrm{~mm}$ DOI $(p<0.001)$. No patient had LN metastasis. In addition, all five patients with C3 subgroup tumours (band-like lymphocytic infiltrate growth pattern) were free of LN metastasis and had not $\geq 3$ LVSI foci.

Given that all GACs had pattern $\mathrm{C}$ invasion, we focused on comparing these tumours with pattern C U-EACs. Morphologically, GACs consisted of tumour cells with distinct cell borders, abundant clear to eosinophilic cytoplasm, and enlarged, irregular and hyperchromatic nuclei. The tumours showed angulated or cystic glands, some with mixed solid, infolded papillae, or cribriform pattern (Fig. 4). Strikingly, all GACs had LVSI, $\geq 3$ LVSI foci, and $>10 \mathrm{~mm}$ DOI. They also 


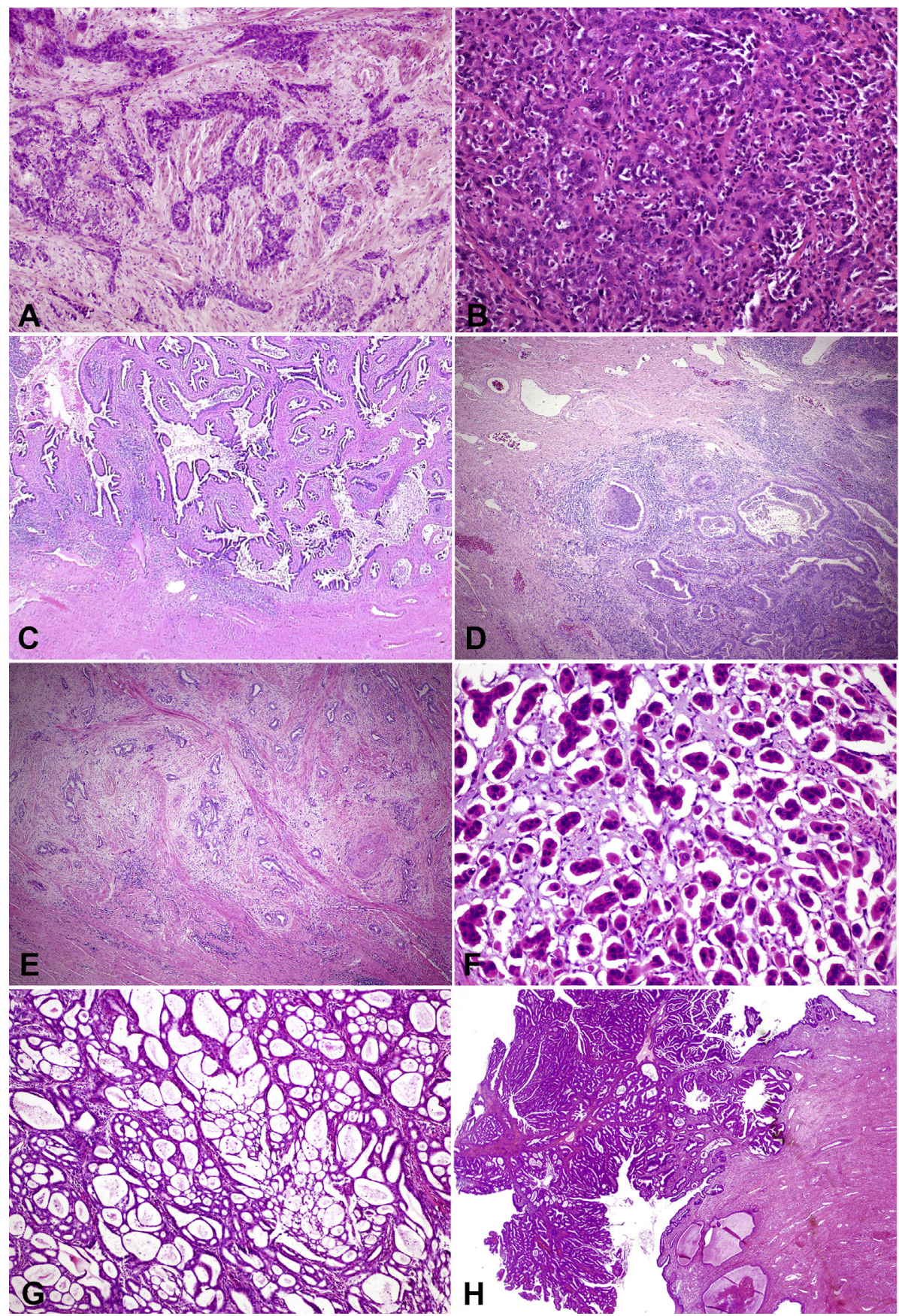

Fig. 3 Pattern C tumours in usual-type endocervical adenocarcinoma. C1 subgroup shows a solid growth pattern with sheets of tumour cells (A) and poorly differentiated components (B). C2 subgroup has an extensive linear destructive growth pattern with invasion at the base of tumour (C). C3 suboroup with band-like lymphocytic infiltrate growth pattern exhibits a prominent lymphoid infiltration in the neoplastic glandular elements (D). C4 subgroup shows diffuse destructive (E), micropapillary $(\mathrm{F})$, or confluent $(\mathrm{G})$ growth pattern. A polypoid tumour has a well-demarcated tumour/stromal interface without destructive stromal invasion, although it exhibits an extensively confluent architecture $(\mathrm{H})$.

had a higher incidence of LN metastasis (66.7\% vs $22.5 \%$, $p=0.001)$ and larger quantity of metastatic LNs [mean 5.5 (2-13) versus mean $2.4(1-12), p=0.004]$ than did U-EACs with pattern $\mathrm{C}$ (Table 3).

LVSI has long been thought to be a prerequisite condition for LN metastasis in carcinoma. In our study, LVSI was identified in 128 cases, corresponding to 1416 slides positive for ADC. The retraction artifact was a common mimic of LVSI and could be identified by IHC analysis for CD34 and D2-40 (Fig. 5) in challenging cases. A total of 4134 LNs from
205 patients were removed [mean 20 (range 4-45)]. Tumour metastasis was observed in $125 \mathrm{LNs}(3.0 \%, 125 / 4134)$ from 42 patients $(20.5 \%, 42 / 205)$. LVSI was detected in all patients with metastatic LNs. To determine whether the quantification of LVSI foci was correlated with LN metastasis, the number of LVSI foci was quantified as 1, 2, 3, 4, 5, 6, 7, 8, 9, and $\geq 10$. Statistical analysis revealed a moderately positive correlation between the number of LVSI foci and status of LN metastasis $(p<0.001, \gamma=0.489)$ or the number of metastatic LNs $(p<0.001, \gamma=0.409)$ (Table 4$)$. 
Table 1 Associations among invasive patterns of endocervical adenocarcinoma and clinicopathological parameters

\begin{tabular}{|c|c|c|c|c|}
\hline Clinicopathological parameters & Pattern A $(n=28)$ & Pattern B $(n=21)$ & Pattern C $(n=164)$ & $p$ value $^{a}$ \\
\hline Mean age at diagnosis, years (range) & $43(26-66)$ & $48(34-67)$ & $49(23-75)$ & 0.007 \\
\hline Usual & $28(100 \%)$ & $21(100 \%)$ & $152(92.7 \%)$ & \\
\hline Gastric & $0(0 \%)$ & $0(0 \%)$ & $12(7.5 \%)$ & \\
\hline No. cases with LVSI (\%) & $0(0 \%)$ & $5(23.8 \%)$ & $123(75.0 \%)$ & $<0.001$ \\
\hline No. LVSI foci $(\%)$ & & & & 0.253 \\
\hline$\geq 3$ & $0(\%)$ & $2(40.0 \%)$ & $80(61.5 \%)$ & \\
\hline No. cases with LN metastases/total cases $(\%)$ & $0 / 24(0 \%)^{\mathrm{b}}$ & $0 / 18(0 \%)^{\mathrm{c}}$ & $42 / 163(25.8 \%)^{\mathrm{d}}$ & $<0.001$ \\
\hline No. metastatic LNs/total LNs (\%) & $0 / 453(0 \%)$ & $0 / 319(0 \%)$ & $125 / 3362(3.7 \%)$ & $<0.001$ \\
\hline DOI $(\%)$ & & & & $<0.001$ \\
\hline$\leq 3 \mathrm{~mm}$ & $20(71.4 \%)$ & $8(38.1 \%)$ & $8(4.9 \%)$ & \\
\hline$>3$ to $\leq 5 \mathrm{~mm}$ & $5(17.9 \%)$ & $6(28.5 \%)$ & $18(11.0 \%)$ & \\
\hline$>5$ to $\leq 10 \mathrm{~mm}$ & $3(10.7 \%)$ & $6(28.5 \%)$ & $32(19.5 \%)$ & \\
\hline$>10 \mathrm{~mm}$ & $0(0 \%)$ & $1(4.8 \%)$ & $106(64.6 \%)$ & \\
\hline
\end{tabular}

DOI, depth of invasion; LN, lymph node; LVSI, lymphovascular space invasion.

${ }^{a} p$ values: age at diagnosis was calculated using ANOVA; other parameters were calculated by the chi-squared test.

$\mathrm{b}$ Two patients underwent hysterectomy without pelvic LN dissection and two were treated by the cone/loop electrosurgical excision procedure.

${ }^{\mathrm{c}}$ Three patients underwent hysterectomy without pelvic LN dissection.

${ }^{\mathrm{d}}$ One patient underwent hysterectomy without pelvic LN dissection.

Table 2 Comparison of the three growth pattern subgroups in usual-type endocervical adenocarcinoma with pattern C

\begin{tabular}{|c|c|c|c|c|c|}
\hline \multirow[t]{2}{*}{ Clinicopathological parameters } & \multicolumn{4}{|c|}{ Usual-type cervical adenocarcinoma with pattern C $(n=152)$} & \multirow[t]{2}{*}{$p$ value $^{\mathrm{a}}$} \\
\hline & $\mathrm{C} 1(n=13)$ & $\mathrm{C} 2(n=15)$ & $\mathrm{C} 3(n=5)$ & $\mathrm{C} 4(n=119)$ & \\
\hline Mean age at diagnosis, years (range) & $47(35-71)$ & $50(35-68)$ & $51(30-75)$ & $49(23-73)$ & 0.920 \\
\hline No. cases with LVSI $(\%)$ & $8(61.5 \%)$ & $3(20.0 \%)$ & $2(40.0 \%)$ & $98(82.4 \%)$ & $<0.001$ \\
\hline No. LVSI foci $(\%)$ & & & & & 0.033 \\
\hline$<3$ & $2(25.0 \%)$ & $3(100 \%)$ & $2(100 \%)$ & $36(36.7 \%)$ & \\
\hline No. cases with LN metastases/total cases $(\%)$ & $4 / 13(30.8 \%)$ & $0 / 15(0 \%)$ & $0 / 5(0 \%)$ & $30 / 118(25.4 \%)^{\mathrm{b}}$ & 0.075 \\
\hline No. metastatic LNs/total LNs (\%) & $8 / 251(3.2 \%)$ & $0 / 333(0 \%)$ & $0 / 82(0 \%)$ & $73 / 2481(2.9 \%)$ & 0.005 \\
\hline Mean no. metastatic LNs (range) & $2.0(1-3)$ & 0 & 0 & $2.4(1-12)$ & $0.700^{\mathrm{c}}$ \\
\hline DOI $(\%)$ & & & & & $<0.001$ \\
\hline$\leq 3 \mathrm{~mm}$ & $2(15.4 \%)$ & $0(0 \%)$ & $1(20.0 \%)$ & $5(4.2 \%)$ & \\
\hline$>3$ to $\leq 5 \mathrm{~mm}$ & $4(30.8 \%)$ & $4(26.7 \%)$ & $2(40.0 \%)$ & $8(6.7 \%)$ & \\
\hline$>5$ to $\leq 10 \mathrm{~mm}$ & $3(23.0 \%)$ & $11(73.3 \%)$ & $1(20.0 \%)$ & $17(14.3 \%)$ & \\
\hline$>10 \mathrm{~mm}$ & $4(30.8 \%)$ & $0(0 \%)$ & $1(20.0 \%)$ & $89(74.8 \%)$ & \\
\hline
\end{tabular}

DOI, depth of invasion; LN, lymph node; LVSI, lymphovascular space invasion.

${ }^{a} p$ values: age at diagnosis was calculated by ANOVA between groups; mean number of metastatic LNs was calculated by Student's $t$ tests; other parameters were calculated by the Pearson chi-squared test.

${ }^{b}$ One patient underwent hysterectomy without pelvic LN dissection.

${ }^{\mathrm{c}}$ Comparison between subgroups $\mathrm{C} 1$ and $\mathrm{C} 4$.

\section{DISCUSSION}

Recent epidemiological studies show an increasing incidence in ADCs worldwide because of early detection of squamous cell precursors in cervical disease screening programs and improved ability to identify glandular lesions. ${ }^{1,2}$ Conventionally, parameters that determine the FIGO stage and prognosis of patients with ADCs include tumour size, DOI and horizontal spread. The treatment approach for patients with ADC is based on FIGO staging. However, FIGO staging alone is not sufficient to confirm patients who could potentially benefit from an aggressive treatment approach.

According to the 2018 FIGO staging system, stage IA or microinvasive cervical cancers are lesions $<5 \mathrm{~mm}$ in depth and further subdivided into stage IA 1 (DOI $<3 \mathrm{~mm}$ ) and stage IA2 (DOI $\geq 3 \mathrm{~mm}$ and $<5 \mathrm{~mm}$ ). ${ }^{8}$ It is well accepted that a more extensive and crowded glandular growth pattern, and a more common stromal reaction (especially desmoplasia) than the normal endocervical tissues can help to identify ADCs. Typical examples with aforementioned invasive patterns are not difficult to diagnose based on careful examination of their morphological features. However, normal glands of the cervix may have hyperplastic and/or complex growth patterns and even occasionally penetrate into the deeper stroma, and some cancerous glands can 'merge into' the stroma instead of infiltrating without a stromal reaction, making it difficult to determine the presence of an invasion. Moreover, the architectural complexity of normal endocervical glands, polypoid or ulcerated tumours, or the coexistence of invasive adenocarcinoma and AIS can lead to confusion in recognising the end of AIS and the start of early invasive ADC. Given these problems, the reproducibility of DOI measurement is extremely low, especially in early invasive lesions. In many cases, tumour thickness rather than DOI is measured, which leads to inaccurate tumour staging. ${ }^{4}$ 


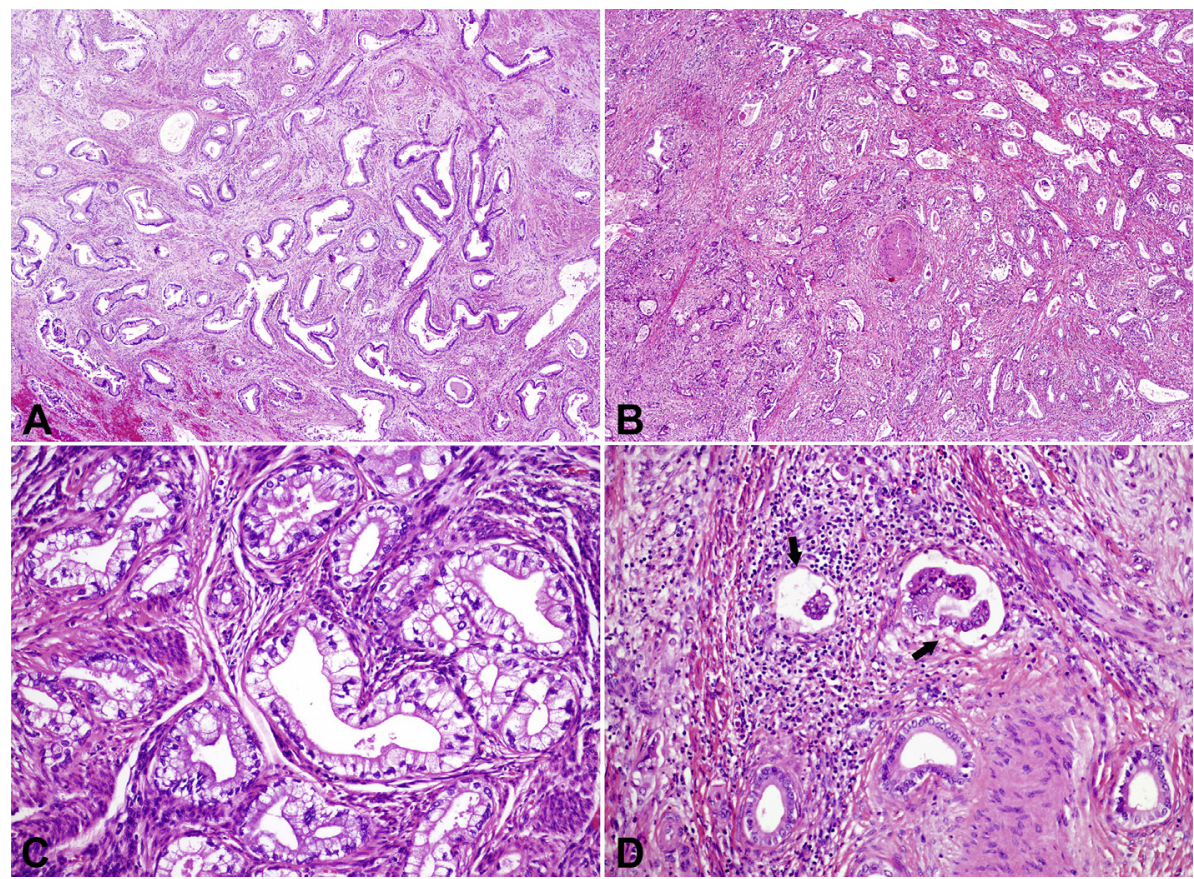

Fig. 4 Gastric-type adenocarcinomas. Neoplastic glands are arranged in a haphazard pattern with prominent stromal desmoplasia (A,B). Tumour cells are characterised by atypical nuclei, abundant pale cytoplasm, and distinct cell borders (C). Foci of lymphovascular space invasion (LVSI; black arrows) and inflammatory infiltration are present (D).

The new Pattern Classification provides a fresh perspective on understanding ADCs. ${ }^{9-11}$ Tumours are classified according to the status (presence or absence) and extent of destructive stromal invasion rather than tumour size and DOI. In line with previous studies, ${ }^{15-17}$ we found that this classification was easy to learn. Pattern A tumours lack aggressive behaviour, because patients do not have LVSI, LN metastasis, and $>10 \mathrm{~mm}$ DOI. Spaans et al. recently proposed that patients with pattern A tumours should receive conservative clinical treatment and be spared lymphadenectomy, the same as patients with AIS, due to an excellent survival, especially in FIGO stage IA. ${ }^{11,17}$ Thus, identifying the AIS lesion from stage IA tumours seems to be less important. A study based on genomic profiles reported that pattern A tumours often lack of oncogenic alterations, whereas pattern $\mathrm{B}$ and pattern $\mathrm{C}$ tumours have multiple oncogenic mutations such as PIK3CA, KRAS, and ERBB2. Pattern A tumours might represent a distinctly indolent subset of ADCs or an early stage in tumour progression. ${ }^{18}$

Despite the high reproducibility of Pattern Classification, the classification of some exophytic tumours remains controversial. ${ }^{15}$ For example, the polypoid tumour shown in Fig. $3 \mathrm{H}$ has a well-demarcated tumour-stromal interface without destructive stromal invasion, but exhibits an extensively confluent architecture. As per Pattern Classification, the polypoid area should be classified as a pattern $\mathrm{C}$ tumour with confluent growth pattern because of confluent glands filling a $4 \times$ field. However, compared with the confluent growth within the polypoid portion, the relatively important part of the tumour is its base, and the area at the base shows pattern A tumour without stromal invasion. Therefore, in our opinion this tumour might need be regarded as pattern A. In our study, neither of two cases with this exophytic polypoid pattern had presence of LVSI and LN metastases. Due to the limited cases, perhaps all examples with this particular pattern need to be further collected and studied as a separate group to determine which pattern is better assigned to them-A versus $C$, which is a critical distinction.

U-EACs with pattern $C$ have various architectural growth patterns, and Alvarado-Cabrero et al. summarised them into six growth patterns. ${ }^{12}$ Among them, all patients with a micropapillary growth pattern had LN metastasis. Conversely, none of the patients with tumours having an extensive linear destructive growth pattern had LN metastasis. Given that there were two patients with micropapillary growth pattern and $75(49.3 \%, 75 / 152)$ with mixed growth pattern in our study (data not shown), we had to stratify all tumours into four subgroups: $\mathrm{C} 1, \mathrm{C} 2$ and $\mathrm{C} 3$ subgroups corresponded to solid, extensive linear destructive, and bandlike lymphocytic infiltrate growth patterns, respectively, and the C4 subgroup included diffuse destructive, confluent, micropapillary, and mixed growth patterns. Our results indicated that these subgroups had significant differences because tumours of the C2 subgroup (extensive linear destructive growth pattern) had the lowest incidence of LVSI among the four subgroups. None of the $\mathrm{C} 2$ subgroup patients had not $\geq 3$ LVSI foci, metastatic LN, and $>10 \mathrm{~mm}$ DOI. Furthermore, all five patients with C3 subgroup tumours (band-like lymphocytic infiltrate growth pattern) were free of LN metastasis and had $\geq 3$ LVSI foci. Hence, it is understandable that the behaviour of tumours with extensive linear destructive and band-like lymphocytic infiltrate growth patterns is less aggressive than that for the other two subgroups. Future studies should focus on identifying extensive linear destructive and band-like lymphocytic infiltrate growth patterns from pattern $\mathrm{C}$ tumours.

The clinical manifestations, morphology, immunophenotype, histogenesis, and prognosis of U-EACs and GACs are different. ${ }^{13,19,20}$ In our study, a comparison of GACs and UEACs with pattern $\mathrm{C}$ revealed that GACs had higher 
Table 3 Comparison of clinicopathological parameters between gastric-type and usual-type endocervical adenocarcinoma with pattern C

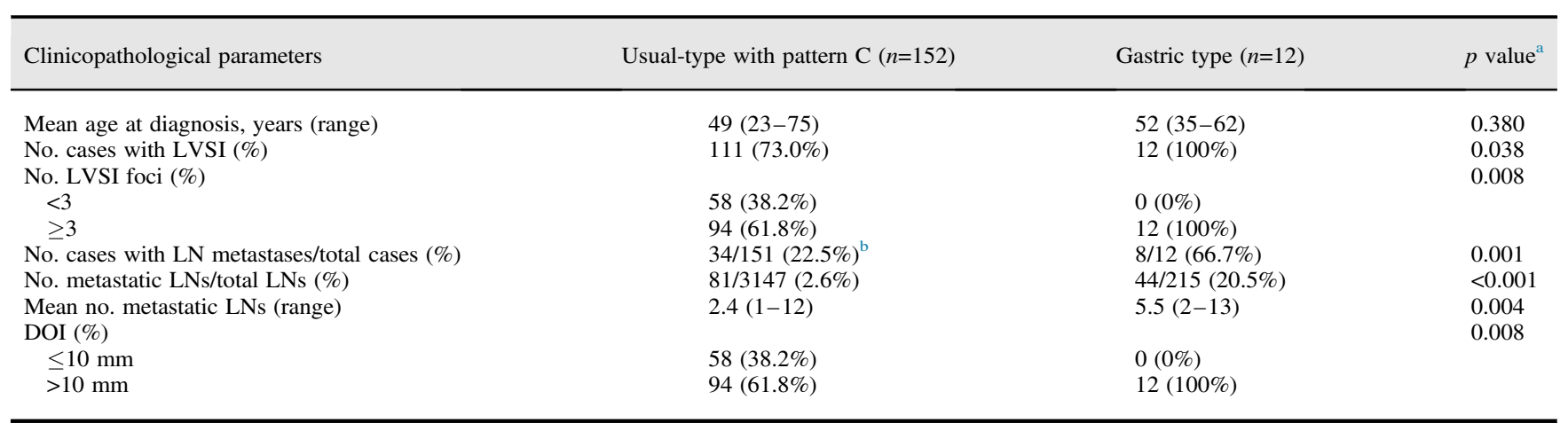

DOI, depth of invasion; LN, lymph node; LVSI, lymphovascular space invasion.

${ }_{b}^{a} p$ values: age at diagnosis and number of metastatic LNs were calculated by Student's $t$ tests; other parameters were calculated by the chi-squared test. ${ }^{\mathrm{b}}$ One patient underwent hysterectomy without pelvic LN dissection.

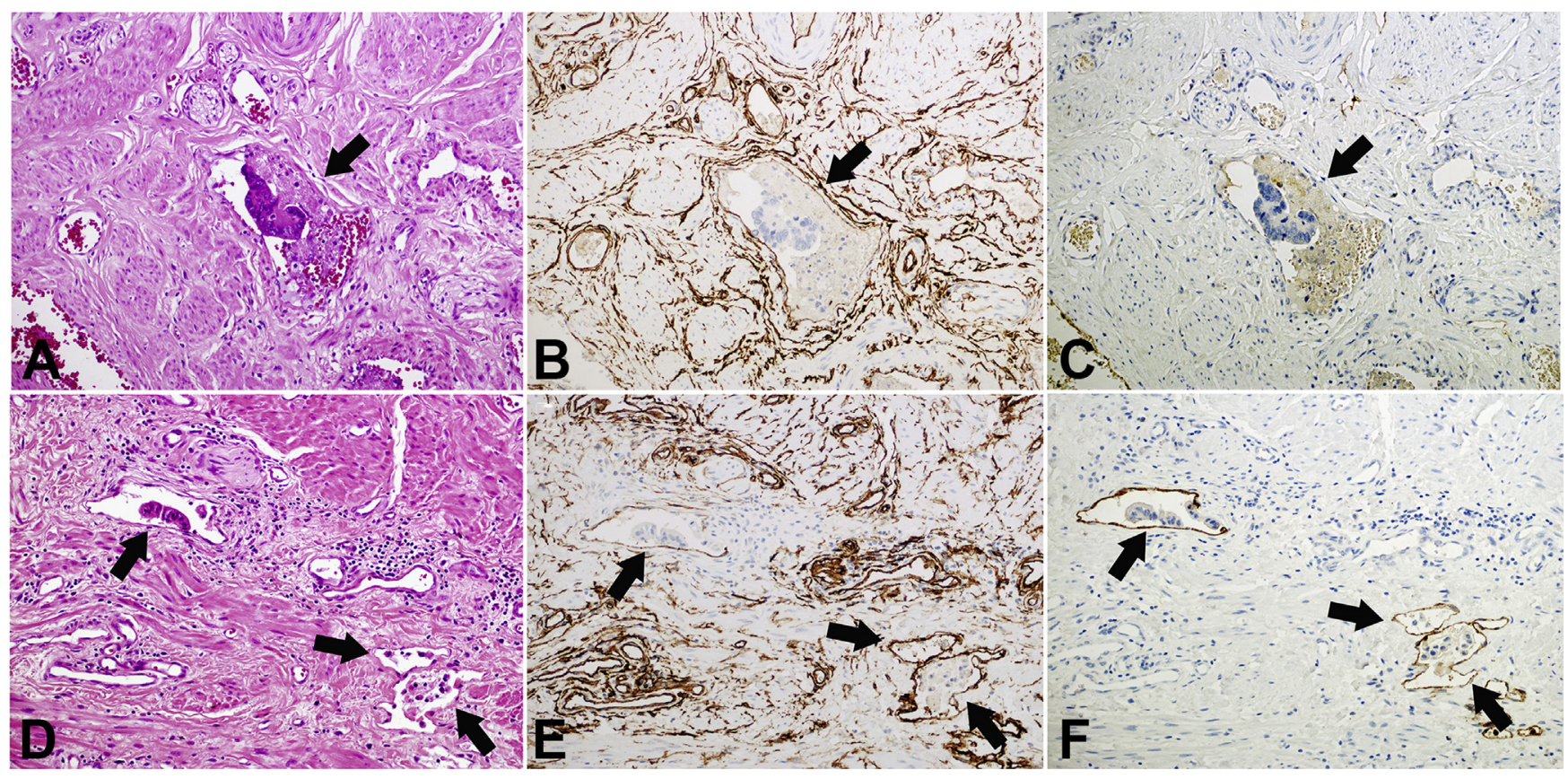

Fig. 5 Lymphovascular space invasion. Tumour embolus in a blood vessel (A) is identified by positive expression of CD34 (B) and negative expression of D2-40 (C). Tumour cells filling the lumen of lymph vessels resemble the retraction artifact (D). Positive CD34 (E) and D2-40 (F) immunostaining confirms the presence of LVSI.

Table 4 Correlation between the quantity of LVSI and status of LN metastasis ${ }^{\mathrm{a}}$

\begin{tabular}{|c|c|c|c|c|}
\hline No. LVSI foci & Total cases $(n)$ & No. cases with LN metastasis ( $n, \%)$ & Metastatic LNs, mean (range) & Total LNs, mean (range) \\
\hline 1 & 20 & $0(0 \%)$ & 0 & $22.50(12-38)$ \\
\hline 3 & 13 & $4(30.8 \%)$ & $0.69(0-4)$ & $17.62(11-45)$ \\
\hline 4 & 10 & $4(40 \%)$ & $0.80(0-3)$ & $22.40(9-36)$ \\
\hline 5 & 6 & $3(50 \%)$ & $0.83(0-2)$ & $19.00(16-26)$ \\
\hline 6 & 7 & $3(42.9 \%)$ & $1.14(0-4)$ & $21.29(8-36)$ \\
\hline 8 & 12 & $5(41.7 \%)$ & $1.75(0-11)$ & $20.33(8-38)$ \\
\hline 9 & 4 & $0(0 \%)$ & 0 & $23.00(17-28)$ \\
\hline$\geq 10$ & 27 & $20(74.1 \%)$ & $2.52(0-13)$ & $21.97(4-28)$ \\
\hline
\end{tabular}

LN, lymph node; LVSI, lymphovascular space invasion.

${ }^{a}$ Correlations between number of LVSI foci and status of LN metastases $(p<0.001, \gamma=0.489)$, and number of metastatic LNs $(p<0.001, \gamma=0.409)$ were calculated using Pearson correlation. 
incidence of LVSI and LN metastasis, deeper stromal invasion, and more LVSI foci and metastatic LNs than did UEACs. These results verify that GAC is an aggressive tumour. However, our study also recommends that GACs should not be analysed by Pattern Classification as they cannot be further risk-stratified. Consistent with our results, a recent study showed that Pattern Classification is only suitable for HPVrelated ADCs. ${ }^{21}$

Studies have provided increasing evidence that the predictive index of Pattern Classification correlates with LVSI, status of LN metastasis, tumour size, FIGO stage, tumour thickness or DOI, perineural invasion, and recurrence-free survival. ${ }^{16,17}$ Invasion pattern as well as tumour size, DOI, LVSI, and LN metastasis have been reported to be independent predictors of recurrence-free and disease-specific survival in a multivariate analysis. ${ }^{21}$ However, whether LN metastasis or LVSI is an independent predictor for overall survival remains controversial. Roma et al. reported that LVSI is not an independent predictor of outcome if LN status is considered. ${ }^{22}$ The behaviour of tumours with both LVSI and metastatic LNs is more aggressive than that for tumours without LVSI and LN metastasis. Thus, the use of Pattern Classification for LVSI and LN status has good predictive value for tumour recurrence and outcome. ${ }^{2}$

Thus far, the correlation between quantitative LVSI and LN metastasis is less known in ADCs. In patients with earlystage squamous carcinoma of the cervix, the quantity of LVSI ( $>45 \%$ of all cervical sections) is an independent predictor of pelvic LN metastasis. However, there is no statistically significant difference between pelvic LN metastasis and the total number of LVSI foci (cut-off point $5){ }^{24}$ Chernofsky et al. reported that the percentage of all sections with LVSI $>29 \%$ and total number of foci with LVSI > 5 is significantly related to tumour recurrence. ${ }^{25}$ Quantifying LVSI by the percentage of cervical sections can yield further useful information on pelvic LN status. Alvarado-Cabrero et al. documented that in ADCs, the quantity of LVSI $>20$ is an independent predictor of LN metastasis. ${ }^{12}$ In our study, only $32.8 \%(42 / 128)$ of patients had both metastatic LN and LVSI foci, although LVSI was detected in all patients with $\mathrm{LN}$ metastasis. There was a moderately positive correlation between the quantity of LVSI and the status of LN metastasis $(p<0.001, \gamma=0.489)$ or quantity of metastatic LNs $(p<0.001, \gamma=0.409)$. Hence, further quantitative information on LVSI, such as the percentage of LVSI in all sections, percentage of all sections with LVSI, maximum number of foci of LVSI per 10 highpower fields, and the number of tumour cells in the largest tumour embolus might be examined in future studies.

In summary, the Pattern Classification provides a valuable method to evaluate ADCs. Pattern $C$ tumours have an aggressive behaviour and are associated with high risk of LVSI and LN metastasis. Patients with tumours with extensive linear destructive and band-like lymphocytic infiltrate growth patterns in Pattern C U-EACs are associated with a lower risk of LVSI and LN metastasis than patients in the other two growth pattern subgroups. However, this classification is not suitable for GACs, because they all belong to pattern $\mathrm{C}$ tumours and cannot be further risk-stratified. Our study suggests that Pattern Classification should be applied routinely to guide therapeutic approaches for patients with UEACs.
Conflicts of interest and sources of funding: Dr Zhang's work was supported by grants from National Natural Science Foundation of China (No. 81572545 and No. 81372783). Dr Pan's research was supported by the American Lebanese and Syrian Associated Charities (ALSAC). The authors state that there are no conflicts of interest to disclose.

Address for correspondence: Dr Jing Zhang, State Key Laboratory of Cancer Biology, Department of Pathology, Xijing Hospital and School of Basic Medicine, Fourth Military Medical University, No. 169 Changle West Road, Xi'an, Shaanxi, 710032, China. E-mail: jzhang@fmmu.edu.cn

\section{References}

1. Zhou J, Wu SG, Sun JY, et al. The effect of local treatment modalities in patients with early-stage adenocarcinoma of the uterine cervix: a population-based analysis. Int J Surg 2017; 41: 16-22.

2. Kurman RJ, Carcangiu ML, Herrington CS, et al. WHO Classification of Tumours of Female Reproductive Organs. 4th ed. Lyon: IARC Press, 2014.

3. Stolnicu S, Barsan I, Hoang L, et al. International Endocervical Adenocarcinoma Criteria and Classification (IECC): a new pathogenetic classification for invasive adenocarcinomas of the endocervix. Am J Surg Pathol 2018; 42: 214-26.

4. Ronnett BM. Endocervical adenocarcinoma: selected diagnostic challenges. Mod Pathol 2016; 29 (Suppl 1): S12-28.

5. Pirog EC. Cervical adenocarcinoma: diagnosis of human papillomavirus-positive and human papillomavirus-negative tumors. Arch Pathol Lab Med 2017; 141: 1653-7.

6. Karamurzin YS, Kiyokawa T, Parkash V, et al. Gastric-type endocervical adenocarcinoma: an aggressive tumor with unusual metastatic patterns and poor prognosis. Am J Surg Pathol 2015; 39: 1449-57.

7. Kojima A, Mikami Y, Sudo T, et al. Gastric morphology and immunophenotype predict poor outcome in mucinous adenocarcinoma of the uterine cervix. Am J Surg Pathol 2007; 31: 664-72.

8. Bhatla N, Aoki D, Sharma DN, et al. Cancer of the cervix uteri. Int $J$ Gynaecol Obstet 2018; 143 (Suppl 2): 22-36.

9. Diaz De Vivar A, Roma AA, Park KJ, et al. Invasive endocervical adenocarcinoma: proposal for a new pattern-based classification system with significant clinical implications: a multi-institutional study. Int $J$ Gynecol Pathol 2013; 32: 592-601.

10. Roma AA, Diaz De Vivar A, Park KJ, et al. Invasive endocervical adenocarcinoma: a new pattern-based classification system with important clinical significance. Am J Surg Pathol 2015; 39: 667-72.

11. Roma AA, Mistretta TA, Diaz De Vivar A, et al. New pattern-based personalized risk stratification system for endocervical adenocarcinoma with important clinical implications and surgical outcome. Gynecol Oncol 2016; 141: 36-42.

12. Alvarado-Cabrero I, Roma AA, Park KJ, et al. Factors predicting pelvic lymph node metastasis, relapse, and disease outcome in pattern $\mathrm{C}$ endocervical adenocarcinomas. Int J Gynecol Pathol 2017; 36: 476-85.

13. Stolnicu S, Barsan I, Hoang L, et al. Diagnostic algorithmic proposal based on comprehensive immunohistochemical evaluation of 297 invasive endocervical adenocarcinomas. Am J Surg Pathol 2018; 42: 989-1000.

14. Smith HO, Qualls CR, Romero AA, et al. Is there a difference in survival for IA1 and IA2 adenocarcinoma of the uterine cervix? Gynecol Oncol 2002; 85: 229-41.

15. Rutgers JK, Roma AA, Park KJ, et al. Pattern classification of endocervical adenocarcinoma: reproducibility and review of criteria. Mod Pathol 2016; 29: 1083-94.

16. Wang W, Song G, Lin J, et al. Study of the revisited, revised, and expanded Silva pattern system for Chinese endocervical adenocarcinoma patients. Hum Pathol 2019; 84: 35-43.

17. Spaans VM, Scheunhage DA, Barzaghi B, et al. Independent validation of the prognostic significance of invasion patterns in endocervical adenocarcinoma: pattern A predicts excellent survival. Gynecol Oncol 2018; 151: 196-201.

18. Hodgson A, Amemiya Y, Seth A, et al. Genomic abnormalities in invasive endocervical adenocarcinoma correlate with pattern of invasion: biologic and clinical implications. Mod Pathol 2017; 30: 1633-41.

19. Wada T, Ohishi Y, Kaku T, et al. Endocervical adenocarcinoma with morphologic features of both usual and gastric types: clinicopathologic and immunohistochemical analyses and high-risk HPV detection by in situ hybridization. Am J Surg Pathol 2017; 41: 696-705.

20. Stolnicu S, Hoang L, Chiu D, et al. Clinical outcomes of HPVassociated and unassociated endocervical adenocarcinomas categorized 
by the international endocervical adenocarcinoma criteria and classification (IECC). Am J Surg Pathol 2019; 43: 466-74.

21. Stolnicu S, Barsan I, Hoang L, et al. Stromal invasion pattern identifies patients at lowest risk of lymph node metastasis in HPV-associated endocervical adenocarcinomas, but is irrelevant in adenocarcinomas unassociated with HPV. Gynecol Oncol 2018; 150: 56-60.

22. Roma AA, Park KJ, Xie H, et al. Role of lymphovascular invasion in pattern C invasive endocervical adenocarcinoma. Am J Surg Pathol 2017; 41: 1205-11.

23. Djordjevic B, Parra-Herran C. Application of a pattern-based classification system for invasive endocervical adenocarcinoma in cervical biopsy, cone and loop electrosurgical excision (LEEP) material: pattern on cone and LEEP is predictive of pattern in the overall tumor. Int $J$ Gynecol Pathol 2016; 35: 456-66.

24. Roman LD, Felix JC, Muderspach LI, et al. Influence of quantity of lymph-vascular space invasion on the risk of nodal metastases in women with early-stage squamous cancer of the cervix. Gynecol Oncol 1998; 68: $220-5$.

25. Chernofsky MR, Felix JC, Muderspach LI, et al. Influence of quantity of lymph vascular space invasion on time to recurrence in women with early-stage squamous cancer of the cervix. Gynecol Oncol 2006; 100: $288-93$. 